\title{
Electrocautery Needling and the 308-nm Excimer Lamp: A Synergistic Combination for the Treatment of Stable Non-segmental Vitiligo
}

\author{
Lili Yang • Pui Mun Wong • Lingli Yang - Ichiro Katayama • \\ Huali Wu $\cdot$ Huimin Zhang
}

Received: April 4, 2020 / Published online: May 21, 2020

(C) The Author(s) 2020

\begin{abstract}
Introduction: Vitiligo is an acquired chronic depigmentation disorder caused by the destruction of melanocytes. Although various treatments have been proposed for the management of vitiligo, achieving repigmentation and preventing relapse remains challenging. The aim of the study was to evaluate the effectiveness of electrocautery needling (EC needling) as a treatment for stable non-segmental vitiligo and to determine if the effectiveness of this treatment could be enhanced by combining it with the 308-nm excimer lamp (excimer lamp).
\end{abstract}

Methods: Thirty patients with stable non-segmental vitiligo were enrolled in this self-

Digital Features To view digital features for this article go to https://doi.org/10.6084/m9.figshare.12264734.

L. Yang · P. M. Wong · H. Zhang $(\bowtie)$

Department of Dermatology, Shuguang Hospital affiliated with Shanghai University of Traditional Chinese Medicine, Shanghai, China

e-mail: zhanghm@shutcm.edu.cn

L. Yang · I. Katayama

Department of Pigmentation Research and

Therapeutics, Graduate School of Medicine, Osaka

City University, Osaka, Japan

H. Wu

Department of TCM Chemistry, School of

Pharmacy, Shanghai University of Traditional

Chinese Medicine, Shanghai, China controlled, non-blinded study. Three vitiligo lesions of similar size, location and disease duration were selected from each patient and randomly assigned to one of three groups treated weekly with EC needling, an excimer lamp or a combination of both (combination group), respectively. The effectiveness of treatment on the repigmentation percentage and the number of treatments required for initial pigmentation were assessed.

Results: There was no significant difference in the repigmentation percentage between the EC needling group and the excimer lamp group $(P=0.789)$. The mean number of treatments required for initial repigmentation was lower in the EC needling group than in the excimer lamp group $(P=0.049)$. The repigmentation percentage was significantly higher in the combination group than in the EC needling group $(P=0.027)$ and excimer lamp group $(P=0.005)$. Evidence of initial pigmentation was obtained earlier in lesions treated with the combination therapy than in lesions treated with excimer lamp therapy alone $(P=0.019)$. Vitiligo lesions on the face and neck regions showed the highest repigmentation percentage among all anatomical regions, whereas lesions on the hands and feet showed the worst treatment response.

Conclusion: Electrocautery needling monotherapy was effective in treating vitiligo, and its efficacy was enhanced when combined with the 308-nm excimer lamp. This combined 
approach to treat vitiligo is safe and helps increase patient compliance.

Keywords: Electrocautery needling; Repigmentation; Vitiligo; 308-nm excimer lamp

\section{Key Summary Points}

Why carry out the study?

Vitiligo is an acquired chronic depigmentation disorder of the skin resulting from the selective destruction of melanocytes, with a prevalence ranging from 0.2 to $1.8 \%$ of the global population. Treatment is often challenging in terms of repigmentation.

The aim of our clinical study was to evaluate the effectiveness of electrocautery (EC) needling monotherapy as a treatment for stable non-segmental vitiligo and to determine whether its effectiveness could be enhanced when used in combination with the 308-nm excimer lamp.

\section{What was learned from the study?}

EC needling monotherapy was effective in treating vitiligo, and its efficacy was enhanced when combined with a 308-nm excimer light.

This combined approach to treat vitiligo is safe and helps increase patient compliance.

\section{INTRODUCTION}

Vitiligo is an acquired chronic depigmentation disorder of the skin that results from the selective destruction of melanocytes [1], affecting $0.2-1.8 \%$ of the global population [2]. The exact aetiology of vitiligo remains unclear, but autoimmune factors have been strongly implicated in the development of the disease [3]. According to the Vitiligo Global Issues
Consensus Conference, vitiligo is mainly classified into segmental and non-segmental vitiligo based on clinical evidence and is categorized into the progression or stable stage based on the activity of the disease [4]. Disease management in the progression stage is aimed at arresting the development of depigmentation, whereas repigmentation and relapse prevention are the main goals of treatment in the stable stage [5]. The treatment of vitiligo is challenging, with current management strategies including medical, surgical and phototherapy treatments [6]. One phototherapy strategy that has shown potential in terms of promoting repigmentation in vitiligo is the 308-nm excimer lamp (subsequently referred to as the excimer lamp), with white patches showing great improvement following treatment, depending on the treatment site and patient age $[7,8]$. Compared to other methods, the excimer lamp is relatively suitable for promoting repigmentation and for stabilizing the disease by enhancing the migration and proliferation of melanocytes through immunosuppression and immunomodulation $[9,10]$.

In recent years, we have used electrocautery needling (EC needling) to treat vitiligo and have achieved good results. The application of EC needling in vitiligo is inspired by fire needle therapy, which has been used to treat vitiligo for decades in traditional Chinese medicine. In EC needling, an electrical current is used to heat a metal probe that is then applied to the target tissue with the aim to burn or coagulate that specific area of tissue. The method is widely used to treat superficial diseases of the skin and mucous membranes due to its safety, efficiency and low cost. During vitiligo treatment, a handheld therapeutic stylus is used to prick the white spot quickly with the needle tip until the white area is covered with pricking points spaced 3-5 mm apart. A high temperature is generated when the energized needle tip comes into contact with the skin. This high temperature simulates the heat-stabbing damage to the puncture site caused by the burning of the traditional fire needle tip and, subsequently, stimulates the skin to initiate the damage repair mechanism and activate melanocyte regeneration. 
We performed a self-controlled non-blinded study to investigate the effectiveness of EC needling therapy for treating stable non-segmental vitiligo and to determine whether the effectiveness of this therapy could be enhanced when used in combination with the excimer lamp.

\section{METHODS}

\section{Study Design}

We conducted a prospective analysis of patients with non-segmental vitiligo in the stable stage who underwent weekly therapy with EC needling, the excimer lamp (USHIO, Tokyo, Japan) or a combination of both therapies (combination group), respectively, in the Department of Dermatology of Shuguang Hospital affiliated with Shanghai University of Traditional Chinese Medicine from June 2016 to December 2019. Thirty patients with stable non-segmental vitiligo lesions were recruited to this study. All patients had a Fitzpatrick skin phototype ranging from III to IV. The inclusion criteria included patients with vitiligo patches who had no previous treatment for at least 8 weeks and patients who were stable and had had no expansion of patches in the past 6 months. The exclusion criteria included a history of photosensitive disorder, melanoma, autoimmune diseases, psychological disorders, pregnancy, lactation and other skin diseases.

All procedures were approved by the ethics committee of Shuguang Hospital affiliated with Shanghai University of Traditional Chinese Medicine and were in accordance with the Helsinki Declaration of 1964 and its later amendments. Informed consent and consent to publish were obtained from each patient.

\section{Treatment}

Three lesions of similar size, location and disease duration were selected from each patient with stable non-segmental vitiligo, resulting in 30 patients and a total of 90 skin lesions being investigated in this study. These 90 skin lesions were randomly assigned to one of three groups: EC needling group $(n=30)$, excimer lamp group $(n=30)$ and combination group $(n=30)$.

Lesions in the EC needling group were treated with local anaesthetic (compound lidocaine cream; Tsinghua Tongfang, Beijing, China) $60 \mathrm{~min}$ prior to the procedure. The instrument was turned on and adjusted to the lowest output power. The handheld monopolar electrocautery pencil was used to prick the white spot with the needle tip to a depth of approximately $1 \mathrm{~mm}$, moving the needle as quickly as possible over the white spot until it was covered with pricking points spaced $3-5 \mathrm{~mm}$ apart (Fig. 1). The treatment was conducted once a week. The patients were advised to avoid having the treated site come into contact with water on the day after needling and to allow the crust to fall off naturally.

Excimer lamp treatment was conducted once weekly at an initial fluence of $150 \mathrm{~J} / \mathrm{cm}^{2}$ for the face and neck, $200 \mathrm{~J} / \mathrm{cm}^{2}$ for the trunk and extremities and $400 \mathrm{~J} / \mathrm{cm}^{2}$ for the hands and feet. The fluence of subsequent treatments was adjusted, or not, based on the duration of erythema. Patients without erythema were treated with a fluence increase of $50 \mathrm{~mJ} / \mathrm{cm}^{2}$. Mild erythema was regarded to be the optimum treatment effect, and in this case the previous fluence was maintained. If symptomatic erythema persisted for $>48 \mathrm{~h}$, the fluence was decreased by $50 \mathrm{~mJ} / \mathrm{cm}^{2}$. If blistering or burning sensation developed, the treatment was discontinued until resolution and then resumed at a fluence that was $50 \mathrm{~mJ} / \mathrm{cm}^{2}$ lower [11]. Patients and physicians wore protective glasses during the procedure for safety purposes.

In the combination treatment, EC needling was carried out 5-10 min after the excimer lamp treatment, both as described above. No dressings were needed, but the lesional area was cleaned with alcohol prior to needling. All patients were told to avoid exposure to the sun during outdoor activities by covering the vitiligo lesion with necessary clothing throughout the experiment to reduce potential effects of environmental ultraviolet B (UVB) exposure. 

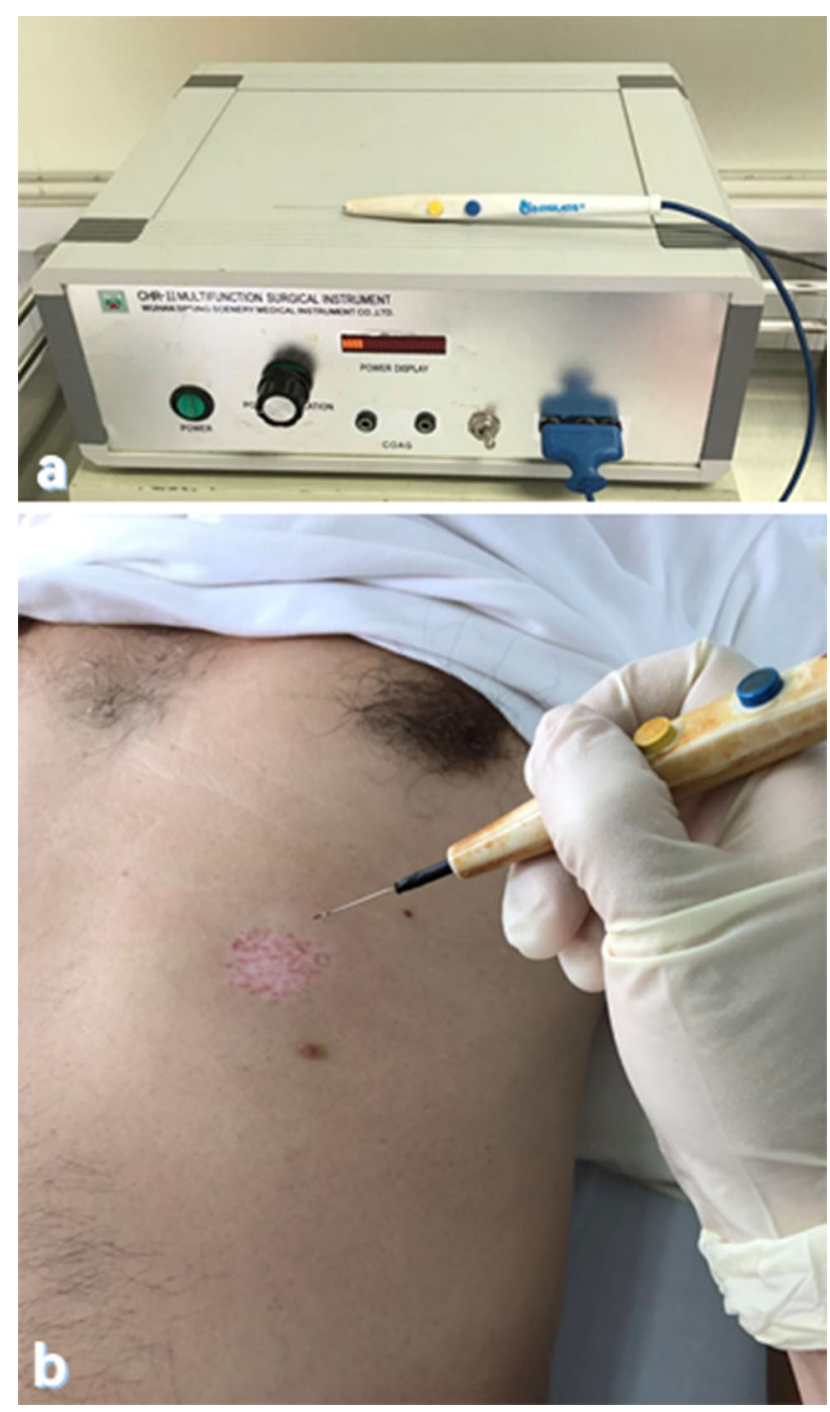

Fig. 1 a Electrocautery instrument, b process of electrocautery needling, showing the handheld monopolar electrocautery pencil

\section{Treatment Response and Efficacy Assessments}

Clinical outcomes were evaluated based on repigmentation percentage after 12 weeks of treatment and on the number of treatments required to obtain follicular or peripheral repigmentation. Body areas, such as the face, trunk, extremities and hands and feet, were grouped according to the location of the lesions to compare the efficacy of treatment by different anatomical regions. Two dermatologists who were not involved in the treatment procedure were assigned to evaluate all photographs taken at the baseline pretreatment visit and posttreatment. Photographs of the skin lesions before treatment and after 12 weeks of treatment were captured by a digital camera with a resolution of 12.0 megapixels. The original area of the lesion and repigmented area in the digital photographs were objectively evaluated by Image-Pro Plus 6.0 software (Media Cybernetics, Rockville, MD, USA). The repigmentation percentage was determined by the formula: (area repigmented/original area with the lesion) $\times 100 \%[12]$. 


\section{Statistical Analysis}

Statistical analysis was performed using SPSS software version 22.0 (IBM Corp., Armonk, NY, USA). One-way analysis of variance was used to evaluate the difference in the repigmentation percentage and the number of treatments for initial pigmentation between the areas treated with EC needling, the excimer lamp, and the combined therapy. Multivariate linear regression analysis was used to determine the correlation between the repigmentation percentage and several confounding factors (age, sex and disease duration) in addition to the treatment intervention. Statistical significance was defined as $P<0.05$.

\section{RESULTS}

\section{Participants}

Thirty patients with stable non-segmental vitiligo were enrolled in the study. Of these, six left the study before study end, with the most common reasons being non-compliance and use of additional medication. Consequently, 24 patients completed the study and were included in the analysis. The baseline demographics and clinical characteristics of those 24 patients who completed the study are given in Table 1.

\section{Repigmentation Percentage According to Groups}

The mean repigmentation percentage of vitiligo lesions in the three treatment groups is presented in Fig. 2. Lesions treated with EC needling achieved a mean repigmentation percentage of $34.86 \% \quad(n=24)$, while those treated with excimer lamps and the combination treatment achieved mean repigmentation percentages of $31.29 \%(n=24)$ and $50.95 \%$ $(n=24)$, respectively. The mean repigmentation percentage was significantly higher in the combination group than in the EC needling group $(P=0.027)$ and excimer lamp group $(P=0.005)$. There were no significant differences in the repigmentation percentage between the EC needling group and excimer lamp group $(P=0.789)$ (Fig. 2$)$. In most cases, repigmentation occurred in perifollicular and peripheral patterns (Fig. 3).

\section{Repigmentation Percentage According to Lesion Location}

As shown in Table 2, the average repigmentation percentage was $50.63 \%$ for patients with lesions on the face $(n=8), 38.70 \%$ for patients with lesions on the trunk $(n=6), 37.01 \%$ for patients with lesions on the extremities $(n=5)$ and $19.69 \%$ for patients with lesions on the hands and feet $(n=5)$. These results show that lesions on the face and neck region responded better to treatment than those on the trunk, extremities, hands and feet, with lesions on the hands and feet showing the worst response.

The repigmentation percentage varied in the three groups according to lesion location (Table 2), being higher for lesions on the face and neck, trunk, extremities and hands and feet treated with the combination therapy than for those treated by the excimer lamp alone and EC needling alone. However, there were no significant differences in repigmentation percentage between the three treatment groups $(P>0.05)$ (Table 2). EC needling showed a greater efficacy in terms of repigmentation percentage in the hands and feet regions than the excimer lamp, but there were no significant differences in the repigmentation percentage between these two treatment groups $(P=0.46)$.

\section{Evaluating the Number of Treatments for Initial Pigmentation in the Three Groups}

In the majority of cases, initial repigmentation occurred between 2 and 10 weeks of treatment initiation. The average number of treatments for initial repigmentation in the EC needling, excimer lamp and combination therapy groups were 4.88, 6.63 and 4.54, respectively (Fig. 4). Evidence of initial pigmentation was obtained earlier in lesions treated with the combination therapy than in those treated with excimer lamp therapy alone $(P=0.019)$. The mean 
Table 1 Demographic data, clinical characteristics and location of the reference lesions at baseline

\begin{tabular}{ll}
\hline Characteristics & Values \\
\hline $\begin{array}{l}\text { Number of patients with stable non- } \\
\text { segmental vitiligo }\end{array}$ & 24 \\
Age (years) & \\
Range & $25-72$ \\
Mean \pm SD & $46.8 \pm 14.1$ \\
Sex $(n)$ & \\
Female & 13 \\
Male & 11 \\
Duration of disease (years) & \\
Range & $1-25$ \\
Mean \pm SD & $6.8 \pm 6.2$ \\
Treated sites, $n(\%)^{a}$ & \\
Head and neck & $5(33.3 \%)$ \\
Trunk & $6(25 \%)$ \\
Extremities & $5(20.8 \%)$ \\
Hands and feet & $5.8 \%)$ \\
\hline
\end{tabular}

$S D$ Standard deviaiton

${ }^{a} n=$ number of patients number of treatments required for initial repigmentation was distinctly lower in the EC needling group than in the excimer lamp group $(P=0.049)$. There were no differences in the treatment number between the EC needling and combination groups ( $P=0.698)$ (Fig. 4).

Multivariable linear regression was performed to determine the correlation between the response of treatment and age, sex, disease duration and lesion location. No correlation was found between the rate of response and age, sex or disease duration.

Some patients in this study who were treated with EC needling experienced varying degrees of pain, measured on a visual analogue scale (VAS: $0-1$ for the face and neck, $0-2$ for the trunk and extremities, and 1-2 for the hands and feet), a burning sensation, erythema and crustation. However, all of these complications disappeared within 1 day, with the exception of crustation, which usually disappeared within 1-2 weeks. Moreover, pricking spots that appeared pale and oedematous $1 \mathrm{~min}$ after needling were considered to be normal and usually disappeared within $30 \mathrm{~min}$. None of the patients treated with EC needling developed infections. Skin lesions exposed to excimer lamp irradiation exhibited different degrees of erythema, and some patients experienced burning sensations and desquamation at the

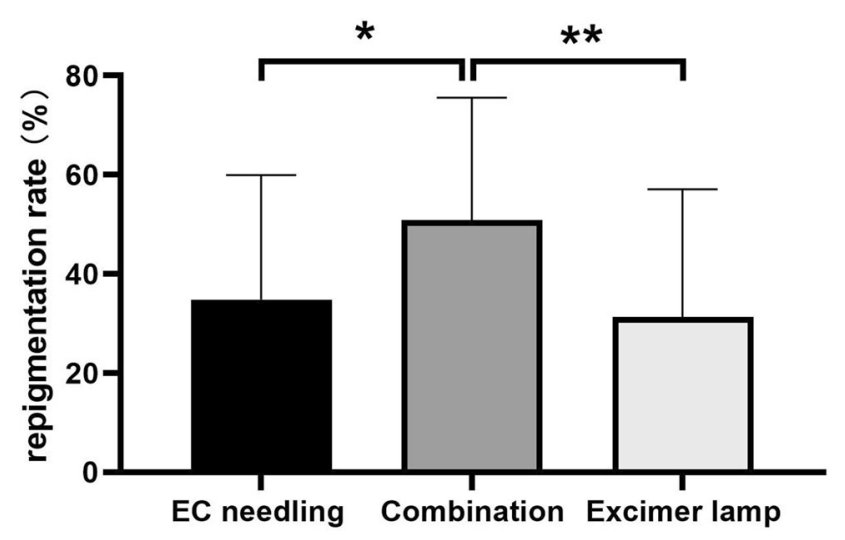

Fig. 2 Mean repigmentation percentage after 12 weeks of treatment in the different treatment groups. Asterisks indicate a significant difference between the indicated groups at ${ }^{*} P<0.05$ and ${ }^{*} P<0.01$. The mean repigmentation percentage was significantly higher in the combination group than in the electrocautery $(E C)$ needling group and excimer lamp group $(n=24$ patients) 

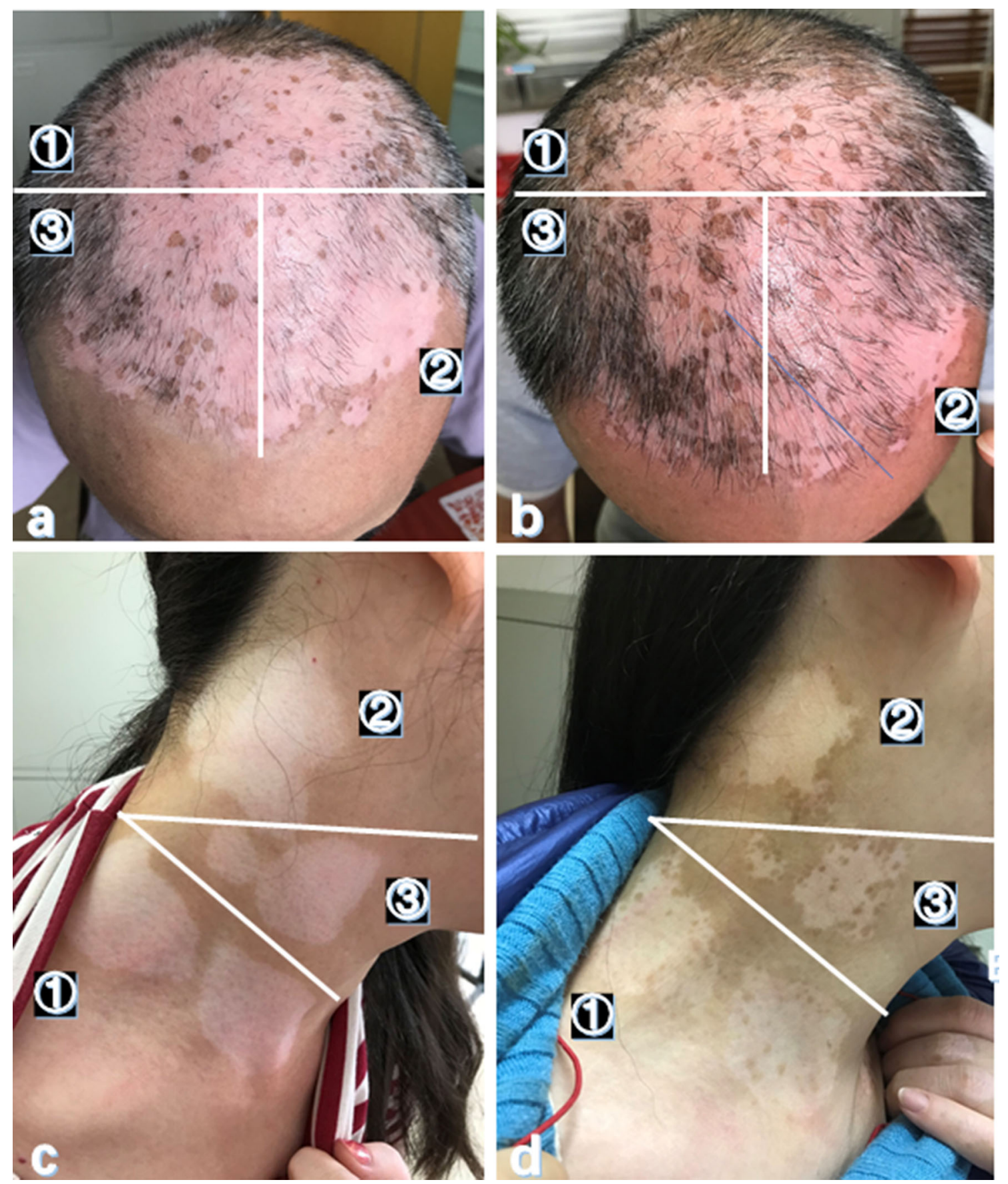

Fig. 3 Baseline (a, c) and posttreatment (b, d) photographs of vitiligo lesions located on the scalp and neck in two patients. Region (1) was treated with EC needling

alone, region (2) was treated with a 308-nm excimer laser lamp alone, and region (3) has been treated with the combination of both therapies 
Table 2 Mean repigmentation percentage according to treatment group and location of the lesions after 12 weeks of treatment

\begin{tabular}{llll}
\hline Location of lesion & \multicolumn{4}{l}{ Repigmentation percentage (minimum-maximum ) after 12 weeks of treatment } \\
\cline { 2 - 4 } & Electrocautery needling group & Excimer lamp group & Combination group \\
\hline Face and neck & $46.95(12.65-78.40)$ & $44.96(15.63-80.34)$ & $66.00(23.45-88.0)$ \\
Trunk & $31.07(12.86-56.68)$ & $32.26(0-78.4)$ & $52.76(22.56-80.55)$ \\
Extremities & $34.34(5.68-75.66)$ & $28.28(10.55-76.26)$ & $48.40(30.66-79.48)$ \\
Hands and feet & $20.60(0-51.22)$ & $11.25(0-23.45)$ & $27.23(0-58.42)$ \\
\hline
\end{tabular}

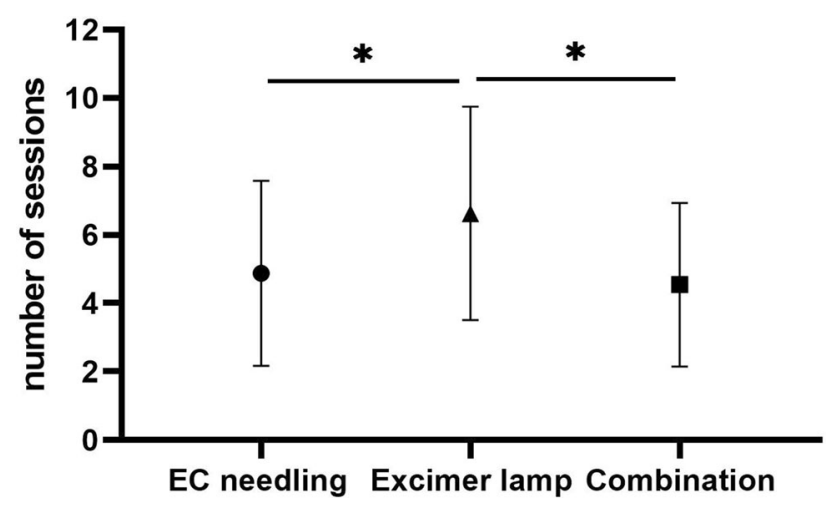

Fig. 4 The number of treatments for initial pigmentation in the three groups. Asterisk indicates a significant difference between the indicated groups at ${ }^{*} P<0.05$

treated lesions which could be relieved by reducing the treatment dose.

\section{DISCUSSION}

The treatment of vitiligo has historically been a therapeutic challenge, and attempts to increase the efficacy of known monotherapies has led to the testing of various combination treatments. The 308-nm excimer lamp was first used to treat vitiligo in 2003, at which time it demonstrated clinical efficacy [13]. Since then, many trials have utilized the lamp for the treatment of vitiligo as monotherapy and as part of combination therapy regimens [14-16]. Different results have been reported in different ethnic populations. In our study, once-weekly excimer lamp treatments induced a $31.29 \%$ repigmentation percentage in 24 patches at 12 weeks posttreatment; over $87.5 \%$ of vitiligo lesions showed different degrees of repigmentation at 4-8 weeks after treatment initiation. Of the lesions treated with the excimer lamp only, mean initial repigmentation occurred at 6.6 treatment sessions, which was later than in those treated with the combination therapy. Combination therapies are therefore widely applied in clinical practice to increase the effectiveness of excimer phototherapy and reduce treatment time.

Fire needle therapy originated from traditional Chinese medicine and has been used for millennia as an alternative treatment for various diseases and maladies. In recent decades, fire needle therapy has been reported as a treatment of vitiligo and has achieved good results [17]. The mechanism of repigmentation is speculated to be related to the inflammation induced by fire needle therapy, which might stimulate the migration of melanocytes to the depigmented area [18-21]. However, the 
diameters of traditional fire needles are $>$ $0.6 \mathrm{~mm}$, which easily causes pain and scarring. The needle also needs to be burned in the flame of an alcohol burner lamp before puncture, which is time- and labour-consuming. If the operation is not performed carefully, there may be hidden risks of burns and fire. These drawbacks limit the applications of fire needles in vitiligo treatment.

The disadvantages of fire needle therapy inspired us to replace this method with electrocautery instruments. Electrocautery is driven by electric energy, and the needling tip can be instantly heated to high temperature and maintained at constant temperature, which makes it possible to puncture lesions continuously. This technological improvement gives EC needling many advantages over traditional fire needle therapy, such as less time intensive, easy to perform and lower costs. In addition, the finer needling tip reduces the pain caused by pricking and improves patient compliance. We have previously compared the effect of EC needling and traditional fire acupuncture in the treatment of vitiligo and found a similar efficacy in both therapies regarding the inducation of repigmentation (data not shown).

In our study, the efficacy of EC needling was comparable to that of the excimer lamp, with lesions treated with the excimer lamp and and those treated with EC needling achieving a similar repigmentation percentage (Fig. 2). However, the EC needling group required fewer treatment sessions than the excimer lamp group for initial repigmentation (Fig. 4), which indicates that EC needling was faster in terms of initiating repigmentation than the excimer lamp. Surprisingly, our data showed that the repigmentation percentage of vitiligo lesions in the combination group was significantly higher than that in the EC needling group $(P=0.027)$ or excimer lamp group $(P=0.005)$, and the number of treatments required for initial repigmentation was significantly lower in the combination group than in the excimer lamp group $(P=0.019)$. These results suggest that the combination treatment was more effective in initiating and achieving excellent repigmentation than excimer lamp monotherapy. In addition, combination therapy could reduce the side effects of excimer lamps, such as photosensitivity, photoaging and photocarcinogenesis.

We postulate that the mechanisms of repigmentation induced by excimer lamps following EC needling are as follows. The various types of cytokines and growth factors secreted during the wound healing process after EC needling therapy may play a role in mitosis in melanocytes. Recent studies have proposed that wounding induces melanocyte regeneration at the site of injury via the Wnt signalling pathway [22], and the migration of melanoblasts and melanocytes towards the site of injury has been observed under live imaging [20]. In addition, stimulation of hyperpigmentation by burn injury in response to inflammation has been reported [23]. However, the mechanisms involved in the increased effectiveness of excimer lamps when combined with EC needle therapy are still poorly understood, and further investigations are required.

Some of the patients in this study who were treated with EC needling experienced slight pain, burning sensations, erythema and crustation. However, all of these complications disappeared within 1 day, except for crustation, and this adverse effect usually disappeared within 1-2 weeks. Other complications, such as infection, scarring, the Koebner phenomenon, and the aggravation of vitiligo lesions, were not observed in this study. Despite encouraging results, additional studies are required in a larger series of patients to adapt the protocol and to confirm the results.

\section{Limitations}

This study was limited by the small population and was conducted in a non-blinded manner with a short-term follow-up period. Hence, further research should be carried out by using a long-term, double-blinded clinical trial with a larger sample size to confirm the advantage of the combination treatment over the excimer lamp as monotherapy. 


\section{CONCLUSIONS}

Our study showed that EC needling monotherapy was effective in treating vitiligo. This efficacy was enhanced by the combination with 308-nm excimer light. This combined approach to treat vitiligo appears to be safe and helps to increase patient compliance. It is also cost effective and beneficial in different parts of the body. Further research conducted with a double-blinded design and a large population is needed to confirm these results.

\section{ACKNOWLEDGEMENTS}

The authors thank all of the patients who participated in this study and all other members of the study team at the Department of Dermatology, Shuguang Hospital affiliated with Shanghai University of Traditional Chinese Medicine.

Funding. This study and the journals' Rapid Service Fee were funded by the High-Level University Summit Project (Huimin Zhang, Summit Plateau Team), the National Natural Science Foundation of China (81972932), the Shanghai Municipal Health Committee Traditional Chinese Medicine Technology Promotion Project (zyjx-2017005), the Three-Year Action Plan to Further Speed Up the Development of Traditional Chinese Medicine in Shanghai, Construction and Cultivation Project of Dominant Diseases of Traditional Chinese Medicine (ZY(2018-2020)-ZYBZ-10).

Authorship. All named authors meet the International Committee of Medical Journal Editors (ICMJE) criteria for authorship for this article, take responsibility for the integrity of the work as a whole, and have given their approval for this version to be published.

Disclosures. Li-li Yang, Pui-Mun Wong, Ling-li Yang, Hua-li Wu, Ichiro Katayama, and Hui-min Zhang have nothing to disclose.
Compliance with Ethics Guidelines. All procedures performed in studies involving human participants were in accordance with the ethical standards of Shuguang Hospital affiliated with Shanghai University of Traditional Chinese Medicine research committee and with the 1964 Declaration of Helsinki and its later amendments or comparable ethical standards. Informed consent and consent to publish was obtained from all individual participants included in the study.

Data Availability. The datasets generated and analysed during the current study are available from the corresponding author upon reasonable request.

Open Access. This article is licensed under a Creative Commons Attribution-NonCommercial 4.0 International License, which permits any non-commercial use, sharing, adaptation, distribution and reproduction in any medium or format, as long as you give appropriate credit to the original author(s) and the source, provide a link to the Creative Commons licence, and indicate if changes were made. The images or other third party material in this article are included in the article's Creative Commons licence, unless indicated otherwise in a credit line to the material. If material is not included in the article's Creative Commons licence and your intended use is not permitted by statutory regulation or exceeds the permitted use, you will need to obtain permission directly from the copyright holder. To view a copy of this licence, visit http:// creativecommons.org/licenses/by-nc/4.0/.

\section{REFERENCES}

1. Ezzedine K, Eleftheriadou V, Whitton M, van Geel N. Vitiligo. Lancet. 2015;386(9988):74-84.

2. Zhang Y, Cai Y, Shi M, et al. The prevalence of vitiligo: a meta-analysis. PLoS ONE. 2016;11(9): e0163806.

3. Alkhateeb A, Fain PR, Thody A, Bennett DC, Spritz RA. Epidemiology of vitiligo and associated 
autoimmune diseases in caucasian probands and their families. Pigment Cell Res. 2003;16(3):208-14.

4. Ezzedine K, Lim HW, Suzuki T, et al. Revised classification/nomenclature of vitiligo and related issues: the Vitiligo Global Issues Consensus Conference. Pigment Cell Melanoma Res. 2012;25(3): E1-13.

5. Bishnoi A, Parsad D. Clinical and molecular aspects of vitiligo treatments. Int J Mol Sci. 2018;19(5): 1509.

6. Nahhas AF, Braunberger TL, Hamzavi IH. Update on the management of vitiligo. Skin Ther Lett. 2019;24(3):1-6.

7. Ostovari N, Passeron T, Zakaria W, et al. Treatment of vitiligo by 308-nm excimer laser: an evaluation of variables affecting treatment response. Lasers Surg Med. 2004;35(2):152-6.

8. Sun Y, Wu Y, Xiao BH, et al. Treatment of 308-nm excimer laser on vitiligo: a systemic review of randomized controlled trials. J Dermatol Treat. 2015;26(4):347-53.

9. Zhang B, Li T, Tang Y, et al. The effects of 308-nm excimer laser on the infiltration of CD4+, CD8+ T-cells, and regulatory $\mathrm{T}$ cells in the lesional skin of patients at active and stable stages of nonsegmental vitiligo. J Dermatol Treat. 2019;11:1-5.

10. Pacifico A, Leone G. Photo(chemo)therapy for vitiligo. Photodermatol Photo. 2011;27(5):261-77.

11. Alhowaish AK, Dietrich N, Onder M, Fritz K. Effectiveness of a 308-nm excimer laser in treatment of vitiligo: a review. Laser Med Sci. 2013;28(3): 1035-41.

12. Verma G, Varkhande SR, Kar HK, Rani R. Evaluation of repigmentation with cultured melanocyte transplantation (CMT) compared with non-cultured epidermal cell transplantation in vitiligo at 12th week reveals better repigmentation with CMT. J Investig Dermatol. 2015;135(10):2533-5.

13. Leone G, Iacovelli P, Paro Vidolin A, Picardo M. Monochromatic excimer light $308 \mathrm{~nm}$ in the treatment of vitiligo: a pilot study. J Eur Acad Dermatol Venereol. 2003;17(5):531-7.
14. Li LL, Hong JQ, Lan LZ, et al. The effectiveness of combination therapy with 308-nm excimer laser in vitiligo in Han Chinese People. Aust J Dermatol. 2019;60(1):E85-6.

15. Oh SH, Kim T, Jee H, Do JE, Lee JH. Combination treatment of non-segmental vitiligo with a 308-nm xenon chloride excimer laser and topical highconcentration tacalcitol: a prospective, single-blinded, paired, comparative study. J Am Acad Dermatol. $2011 ; 65(2): 428-30$.

16. Passeron T, Ostovari N, Zakaria W, et al. Topical tacrolimus and the 308-nm excimer laser-a synergistic combination for the treatment of vitiligo. Arch Dermatol. 2004;140(9):1065.

17. Wang T, Ren Y, Di D, Zhu Q, Luo G. Segmental vitiligo treated by fire needle therapy: a case series. Eur J Dermatol. 2018;28(1):118-9.

18. Yuan JP, Chen HQ, Yan R, et al. Fractional $\mathrm{CO}_{2}$ lasers contribute to the treatment of stable nonsegmental vitiligo. Eur J Dermatol. 2016;26(6): 592-8.

19. Regazzetti C, Alcor D, Chignon-Sicard B, Passeron T. Micro holes for delivering melanocytes into the skin: an ex vivo approach. Pigment Cell Melanoma R. 2016;29(4):481-3.

20. Levesque M, Feng Y, Jones RA, Martin P. Inflammation drives wound hyperpigmentation in zebrafish by recruiting pigment cells to sites of tissue damage. Dis Model Mech. 2013;6(2):508-15.

21. Chou WC, Takeo M, Rabbani P, et al. Direct migration of follicular melanocyte stem cells to the epidermis after wounding or UVB irradiation is dependent on Mc1r signaling. Nat Med. 2013;19(7): 924.

22. Sun Q, Rabbani P, Takeo M, et al. Dissecting Wnt signaling for melanocyte regulation during wound healing. J Investig Dermatol. 2018;138(7): 1591-600.

23. Dai NT, Chang HI, Wang YW, et al. Restoration of skin pigmentation after deep partial or full-thickness burn injury. Adv Drug Deliv Rev. 2018;123: 155-64. 